\title{
Efetividade de uma intervenção na promoção de estilo de vida de servidores públicos
}

\section{Effectiveness of an intervention in promoting the lifestyle of public servants}

\section{AUTORES \\ Paulo Vitor de Souza ${ }^{1}$ (D) \\ Camila Tomicki ${ }^{1}$ iD \\ Lisandra Maria Konrad ${ }^{1}$ (DD \\ Paula Fabricio Sandreshi ${ }^{1}$ (D) \\ Cezar Grontowski Ribeiro ${ }^{1,2}$ (D) \\ Elaine Cristina Maciel $^{1}$ (D) \\ Cassiano Ricardo $\operatorname{Rech}^{1}$ (iD \\ Tânia R. Bertoldo Benedetti ${ }^{1}$ (iD \\ 1 Universidade Federal de Santa Catarina, Departamento de Educação Física, Florianópolis, Santa Catarina, Brasil. \\ 2 Instituto Federal do Paraná, Departamento de Educação Física, Palmas, Paraná, Brasil.}

\section{CONTATO}

Paulo Vitor de Souza

paulosouzaedf@outlook.com

Universidade Federal de Santa Catarina

Centro de Desportos, Campus Reitor João

David Ferreira Lima, s/n, Trindade,

Florianópolis, Santa Catarina, Brasil.

CEP: 88040-900.

DOI

$10.12820 /$ rbafs. $25 \mathrm{e} 0135$

\section{(cc) BY}

Este trabalho está licenciado com uma Licença Creative Commons - Atribuição 4.0 Internacional.

\begin{abstract}
RESUMO
Este estudo teve por objetivo avaliar o alcance e a efetividade do programa de mudança de comportamento denominado "Vida Ativa Melhorando a Saúde" - VAMOS (versão 2.0) em servidores públicos técnico-administrativos da Universidade Federal de Santa Catarina (UFSC). Trata-se de um estudo de intervenção, do tipo quase experimental, realizado com 35 servidores divididos em grupo intervenção $(\mathrm{GI}=22)$ e controle $(\mathrm{GC}=13)$. O GI participou durante seis meses da intervenção VAMOS e o GC recebeu informações em um único momento sobre a importância de comportamentos saudáveis. $\mathrm{O}$ alcance e a efetividade da intervenção foram avaliados por meio da ferramenta RE-AIM. Foram realizadas medidas de pré e pós-intervenção para avaliar o nível de atividade física, o comportamento alimentar, a antropometria e a qualidade de vida. Para a análise dos dados foi utilizado o teste qui-quadrado, análise de variância two way para medidas repetidas e método de equações de estimações generalizadas $(\mathrm{p}<0,05)$. As análises dos dados foram realizadas no programa IBM $^{\circledR}$ versão 22.0. O alcance da intervenção foi de 14,5\%. Em relação a efetividade, o GI diminuiu o tempo gasto em comportamento sedentário e aumentou a atividade física de intensidade moderada/vigorosa, elevou o consumo de alimentos saudáveis, reduziu as medidas antropométricas e melhorou a percepção da qualidade de vida positiva. Por meio de orientações educacionais sobre a importância da adesão de comportamentos saudáveis relacionados a atividade física e alimentação, o VAMOS promoveu mudanças de comportamentos que produziram impactos positivos na saúde dos servidores públicos técnico-administrativos.
\end{abstract}

Palavras-chave: Saúde do trabalhador; Promoção da saúde; Atividade motora; Dieta saudável; Educação em saúde.

\begin{abstract}
This study aimed to evaluate the reach and effectiveness of the behavior change program called "Active Life Improving Health" - VAMOS (version 2.0) in technical-administrative public servants at the Federal University of Santa Catarina (UFSC). It is an intervention study, of an almost experimental type, carried out with 35 servers divided into an intervention group $(I G=22)$ and a control group $(C G=13)$. The $I G$ participated for six months in the intervention, and the $C G$ received information in a single moment about the importance of healthy behaviors. The reach and effectiveness of the intervention were assessed using the $R E-A I M$ tool. Pre-and post-intervention measures were taken to evaluate the level of physical activity, eating behavior, anthropometry and quality of life. For data analysis, the chi-square test, two-way anal$y$ sis of variance for repeated measures and generalized estimation equation method were used ( $p<0.05)$. The data was analyzed using the $I B M^{\circledR}$ program version 22.0. The reach of the intervention was $14.5 \%$. Regarding effectiveness, the IG decreased the time spent on sedentary behavior and increased the moderatel vigorous physical activity, increased consumption of healthy foods, reduced anthropometric measurements and improved the perception of a positive quality of life. Through educational guidelines on the importance of adhering to healthy behaviors related to physical activity and food, VAMOS promoted behavioral changes which produced positive impacts on the health of technical-administrative public servants.
\end{abstract}

Keywords: Occupational health; Health promotion; Motor activity; Healthy diet; Health education.

\section{Introdução}

As altas prevalências de Doenças Crônicas Não Transmissíveis $(\mathrm{DCNTs})^{1}$ geram impactos negativos aos sistemas de saúde. Neste sentido, é necessário investir em programas e políticas eficazes para reduzir os riscos para a saúde e melhorar a qualidade de vida da população ${ }^{2}$. Estratégias de promoção de saúde tornam-se fundamentais nos locais de trabalho, levando em con- ta o longo período que as pessoas se encontram nestes ambientes $^{3}$. De acordo com a Organização Mundial da Saúde (OMS) e a Organização Internacional do Trabalho (OIT), até 1,2 milhão de mortes por ano no mundo poderiam ser evitadas com locais de trabalho mais saudáveis e seguros ${ }^{4}$.

No Brasil, as principais causas para o absenteísmo por doença em servidores públicos são relacionadas a 
transtornos mentais ou comportamentais e doenças osteomusculares ${ }^{5,6}$. Este fato pode ser reflexo do estilo de vida desses trabalhadores. Em função da sua dinâmica de trabalho, sua rotina inclui atividades de baixa mobilidade que geralmente ocasionam um comportamento sedentário, condição que os predispõe ao risco aumentado para o acometimento de diversos tipos de doenças $^{7-9}$. Aliado a isso, o comportamento alimentar, muitas vezes não é saudável, o que pode agravar a situação de saúde ${ }^{10}$. Comumente, servidores públicos são submetidos a regras formais rígidas, cujas características podem ser importantes no processo saúde-doença e há uma carência de ações institucionais que orientem e/ou incentivem cuidados com a saúde 5 . Esse conjunto de fatores pode ser determinante nas questões de aumento do custo com o absenteísmo dos servidores, já que sua ocorrência ocasiona gastos ineficientes e diminui a qualidade dos serviços prestados ${ }^{11}$.

Dessa forma, é evidente a necessidade de ações que visem mudar o estilo de vida dos trabalhadores. Neste sentido, o "Vida Ativa Melhorando a Saúde" (VAMOS) é um programa de mudança de comportamento que tem por objetivo motivar as pessoas a adotarem um estilo de vida ativo e saudável com relação à atividade física, e alimentação ${ }^{12}$. O VAMOS promove conhecimentos que possibilitam aos seus participantes identificarem possíveis barreiras para adesão da prática de atividade física, bem como reduzir o tempo em comportamento sedentário e manutenção de estilos de vida ativos e saudáveis. Além disso, auxilia no desenvolvimento de estratégias individuais para resolver e minimizar tais barreiras ${ }^{13}$.

Diante disso, considerando a importância do ambiente de trabalho na prevenção de doenças e promoção de saúde, este estudo teve por objetivo avaliar o alcance e a efetividade do Programa VAMOS (versão 2.0) em servidores públicos técnico-administrativos da Universidade Federal de Santa Catarina.

\section{Método}

Trata-se de um estudo de intervenção, do tipo quase experimental, realizado na Pró-Reitoria de Desenvolvimento e Gestão de Pessoas (PRODEGESP) da Universidade Federal de Santa Catarina (UFSC), Campus Florianópolis, Santa Catarina, Brasil. Este foi aprovado pelo Comitê de Ética em Pesquisa com Seres Humanos da UFSC (CAAE no 47789015.8.0000.0121 e parecer $\left.\mathrm{n}{ }^{\circ} 1.394 .492\right)$ e atendeu os preceitos éticos dispostos na Resolução 466/12 do Conselho Nacional de Saúde.
O estudo foi realizado no período de abril a outubro de 2017. Como critério de inclusão, os participantes deveriam ser servidores públicos com cargos técnico-administrativos atuantes nos setores que atuam na PRODEGESP. A técnica de amostragem foi não probabilística por conveniência, uma vez que a própria coordenação da PRODEGESP solicitou a intervenção aos servidores. Deste modo, todos os servidores foram convidados para participarem do Programa VAMOS (versão 2.0) por meio de endereço eletrônico institucional e carta-convite impressa.

Os servidores interessados participaram de uma reunião com membros da equipe do VAMOS que explicaram sobre o protocolo da intervenção e avaliações previstas. Esses servidores constituíram o grupo intervenção (GI). Adicionalmente, um grupo controle (GC) foi composto com os servidores que não participaram da intervenção, sendo selecionados por conveniência.

O GI participou da intervenção durante seis meses, sendo o primeiro mês em encontros semanais e outros cinco meses em encontros quinzenais de aproximadamente 90 minutos cada. Um total de 12 encontros foram realizados nas dependências da UFSC e foram conduzidos por profissionais da saúde - da área de Educação Física e Nutrição, membros da equipe do VAMOS. Os encontros foram realizados em forma de "rodas de conversa" e cada servidor recebeu o material didático impresso que contemplava 12 cadernos (um entregue a cada encontro) com diferentes temas, entre eles: comportamento sedentário, atividade física, alimentação saudável, autoeficácia e apoio social para um estilo de vida ativo e saudável, controle do estresse, administração do tempo, entre outros. Os servidores foram liberados para participar da intervenção no horário de expediente. O GC, por sua vez, recebeu em um único momento nformações breves (aproximadamente 10 minutos), por meio de palestra, sobre a importância de comportamentos saudáveis relacionados à prática de atividade física e alimentação. Essas informações foram oferecidas antes da coleta de pré-intervenção.

A ferramenta RE-AIM ${ }^{14}$ foi usada para avaliar o Programa VAMOS considerando as dimensões de alcance e efetividade, ambas avaliadas em nível individual. O alcance se refere ao número de participantes, proporção da população-alvo que participa e representatividade dos participantes com relação à população-alvo. A taxa referente ao alcance da intervenção foi obtida por meio da divisão do número de servidores que participaram da intervenção pelo número total de 
servidores, multiplicado por 100 .

A efetividade se refere ao quanto a intervenção produziu o resultado esperado (nos desfechos de saúde de interesse). Para isto, a coleta de dados foi centrada na avaliação dos marcadores do Programa VAMOS: primários - atividade física e comportamento alimentar; secundários - antropometria e qualidade de vida. As coletas foram realizadas nos dois grupos (GI e GC) em dois momentos: linha de base (pré-intervenção) e pós-intervenção (após o término dos 6 meses de intervenção).

Dados sociodemográficos foram coletados, por meio de entrevista individual face a face, para identificar as características gerais dos participantes, com dados de idade, sexo, raça, estado civil, escolaridade e renda média mensal. Os participantes também foram questionados sobre suas morbidades e seus estados nutricionais.

As avaliações da atividade física habitual e o comportamento sedentário foram obtidas utilizando acelerômetros da marca Actigraph (GT3X+). Cada servidor foi instruído a utilizar o aparelho no lado direito da cintura durante sete dias consecutivos, retirando-o apenas para dormir, tomar banho ou para realizar atividades aquáticas. Para fins de análise, foram considerados como dados válidos um mínimo de 10 horas de gravações de atividade diária, durante pelo menos quatro dias, sendo três dias de semana e um dia de final de semana. Os dados foram coletados em uma frequência de $30 \mathrm{~Hz}$ e analisados usando epochs de 60s. Períodos com zeros consecutivos durante 60 minutos ou mais (com 2 minutos de tolerância) foram interpretados como tempo de não uso e excluídos da análise ${ }^{15}$. O tempo em comportamento sedentário (CS) $(0-99$ counts min-1), em atividade física leve (AF leve) (100 - 1951 counts min1 ) e em atividade física moderada a vigorosa (AFMV) $(\geq 1952 \text { counts min- } 1)^{16}$ foram calculados por meio do software da ActiLife, ajustando os valores de acordo com o número de dias válidos e horas de uso.

O comportamento alimentar foi avaliado por meio de 6 questões extraídas do inquérito Vigilância de Fatores de Risco para Doenças Crônicas Não-Transmissíveis por Inquérito Telefônico (VIGITEL), do Ministério da Saúde, adaptadas para serem utilizadas como marcadores do Programa ${ }^{17}$. Para este estudo, foram considerados os seguintes alimentos como sendo saudáveis: frutas, verduras/saladas - in natura, verduras/saladas - cozido e feijão; e os seguintes alimentos como não saudáveis: refrigerante, doces e lanches. A partir disso, foi calculado o percentual de participantes que consumia cada item alimentar em 5 ou mais dias da semana.
As variáveis antropométricas foram obtidas por meio de medidas de massa corporal, estatura e circunferência da cintura. As medidas foram coletadas por meio de balança digital da marca Incoterm ${ }^{\circledast}$ com precisão de $0,1 \mathrm{~kg}$, estadiômetro portátil do tipo trena e fita métrica inelástica - ambas da marca Sanny e com precisão de $0,1 \mathrm{~cm}$. Por meio dos escores de estatura e massa corporal foi calculado o índice de massa corporal $(\mathrm{IMC})\left[\mathrm{IMC}=\mathrm{MC}(\mathrm{kg}) / \mathrm{Est}^{2}(\mathrm{~m})\right]$ dos servidores. Os pontos de corte para a circunferência da cintura que foram adotados são os preconizados pela $\mathrm{OMS}^{18}$. Foi utilizado os seguintes valores: risco elevado para mulheres $(\mathrm{CC}>80 \mathrm{~cm})$ e para homens $(\mathrm{CC}>94 \mathrm{~cm})$.

A percepção da qualidade de vida foi avaliada pela questão "Considerando as duas últimas semanas, como você avaliaria a sua qualidade de vida?" do questionário World Health Organization Quality of Life Instrument versão breef(WHOQOL-BREEF), validado para a população brasileira ${ }^{19}$. As opções de resposta eram: "muito ruim", "ruim", "nem ruim nem boa", "boa" e "muito boa". A partir disso, foi calculado o percentual de servidores com qualidade de vida positiva (boa e muito boa).

As análises dos dados foram realizadas no programa $\mathrm{IBM}^{\circledast}$ versão 22.0. Para caracterização da amostra, foram utilizadas análises descritivas usando medidas de frequência absoluta e relativa para variáveis categóricas. O teste qui-quadrado foi utilizado para verificar a homogeneidade nas características entre os servidores no momento pré-intervenção.

Para avaliar a efetividade dos efeitos da intervenção foram realizadas comparações intra e intergrupos para desfechos de atividade física habitual, comportamento alimentar, antropometria e qualidade de vida. As análises foram realizadas por protocolo, ou seja, incluindo apenas os servidores que tiveram os dados coletados nos dois momentos (pré e pós intervenção). Para as variáveis quantitativas (atividade física habitual e antropométricas), aplicou-se a análise de variância bidirecional para medidas repetidas (ANOVA two-way), após confirmação das premissas de esfericidade (teste de Mauchly), com post hoc de Bonferroni, Para as variáveis cuja esfericidade tenha sido violada, conforme indicado pelo teste de Mauchly, as análises foram ajustadas usando-se a correção Greenhouse-Geisser. O método de equações de estimativa generalizadas (GEE) foi aplicado na comparação intra e intergrupos das variáveis de comportamento alimentar e qualidade de vida, analisadas de forma categórica. $\mathrm{O}$ nível de significância adotado foi de 5\%. 


\section{Resultados}

No total de 152 servidores públicos atuavam na PRODEGESP (71,1\% mulheres), no período do estudo, e destes 22 participaram do VAMOS - alcance da intervenção de 14,5\%. Além disso, 13 servidores fizeram parte do CG. Deste modo, um total de 35 servidores participaram do estudo. As características gerais dos servidores estão apresentadas na Tabela 1. Os grupos apresentaram homogeneidade nas características gerais $(\mathrm{p}>0,05)$, com exceção do estado nutricional $(\mathrm{p}<0,01)$.

Tabela 1 - Características gerais dos servidores públicos técnico-administrativos participantes do estudo. Florianópolis, $2017(\mathrm{n}=35)$.

\begin{tabular}{|c|c|c|c|}
\hline \multirow{2}{*}{ Variáveis } & $\mathrm{GI}(\mathrm{n}=22)$ & $\mathrm{GC}(\mathrm{n}=13)$ & \multirow{2}{*}{$\mathrm{p}$ valor } \\
\hline & $\%(\mathrm{n})$ & $\%(\mathrm{n})$ & \\
\hline \multicolumn{3}{|l|}{ Faixa etária } & \multirow{2}{*}{0,092} \\
\hline 20 a 40 anos & $50,0(11)$ & $84,6(11)$ & \\
\hline \multicolumn{4}{|l|}{ Sexo } \\
\hline Mulheres & $77,3(17)$ & $76,9(10)$ & 1,000 \\
\hline \multicolumn{4}{|l|}{ Raça } \\
\hline Brancos & $81,8(18)$ & $84,6(11)$ & 1,000 \\
\hline \multicolumn{4}{|l|}{ Estado civil } \\
\hline Com companheiro & $63,6(14)$ & $61,5(8)$ & 1,000 \\
\hline \multicolumn{4}{|l|}{ Nível educacional } \\
\hline Pós-graduados & $54,5(12)$ & $53,8(7)$ & 1,000 \\
\hline \multicolumn{4}{|l|}{ Renda média mensal } \\
\hline$\geq 6 \mathrm{SM}$ & $68,2(15)$ & $61,5(8)$ & 0,975 \\
\hline \multicolumn{4}{|l|}{ Morbidades } \\
\hline $\operatorname{Sim}$ & $59,1(13)$ & $30,8(4)$ & 0,204 \\
\hline \multicolumn{4}{|l|}{ Estado nutricional } \\
\hline Com sobrepeso/obesidade & $59,1(13)$ & $7,7(1)$ & $0,008^{*}$ \\
\hline \multicolumn{4}{|l|}{ Obesidade abdominal } \\
\hline Sim & $59,1(13)$ & $46,2(6)$ & 0,696 \\
\hline
\end{tabular}

GI = grupo intervenção; $\mathrm{GC}$ = grupo controle; $\mathrm{SM}=$ salário mínimo com base em 2017 - R\$ 937,00; DVC = doença cardiovascular; $\mathrm{n}=$ número de servidores; $\mathrm{p}=$ valor de probabilidade; ${ }^{\mathrm{p}}<0,05=$ Teste Qui-quadrado.

Ao final da intervenção 13 servidores concluíram a mesma, representando uma taxa de retenção de 59,1\%. Alguns dos motivos das desistências foram devido a problemas de saúde $(n=2)$, questões familiares $(n=3)$, mudança de setor de trabalho $(n=1)$, afastamento para cursar mestrado $(n=1)$ e falta de interesse $(n=2)$.

A Tabela 2 apresenta o tempo diário em $\mathrm{CS}, \mathrm{AF}$ leve, AFMV e AF total nos dois grupos e nos momentos pré e pós-intervenção. Houve interação grupo vs. tempo estatisticamente significante indicando que o GC aumentou o tempo gasto em AF leve em comparação ao $\mathrm{GI}(\mathrm{p}=0,042)$. Não houve efeito isolado para o tempo gasto em CS, AFMV e AF total ( $p>0,05)$.

A Tabela 3 apresenta o consumo de alimentos saudáveis e não saudáveis em cinco ou mais dias da semana nos dois grupos, nos momentos pré e pós-intervenção. Em relação ao consumo de alimentos saudáveis, o GI melhorou seu comportamento alimentar em todos os itens alimentares analisados. Interação grupo vs. tempo estatisticamente significante indicou que o GI aumentou somente o consumo de verduras/saladas cozidos enquanto o GC reduziu o consumo ( $\mathrm{p}<0,001)$. Não foi observado efeito isolado para o consumo de frutas, verduras/saladas in natura e feijão $(p>0,05)$. Não houve interação ou efeito isolado para o consumo de alimentos não saudáveis (refrigerante, doces e lanches) ( $p>0,05$ ).

A Tabela 4 apresenta a massa corporal, o índice de massa corporal (IMC) e a circunferência da cintura, nos momentos pré e pós-intervenção. O GI reduziu a massa corporal, o IMC e a circunferência da cintura, enquanto o GC reduziu somente a medida da circunferência da cintura. Não houve interação grupo vs. tempo ( $p>0,05)$. Observou-se efeito isolado do grupo para massa corporal e IMC ( $p=0,022$ e $p=0,036$, respectivamente).

A Tabela 5 apresenta a percepção da qualidade de vida positiva nos dois grupos, nos momentos pré e pós-intervenção. Interação grupo vs. tempo estatisticamente significante indicou aumento no número de participantes do GI com percepção da qualidade de vida positiva após participação no Programa VAMOS $(\mathrm{p}=0,048)$.

\section{Discussão}

Este estudo teve como objetivo avaliar o alcance e a efetividade do Programa VAMOS (versão 2.0) em servidores públicos técnico-administrativos da $\mathrm{PRO}-$ DEGESP da UFSC. Mesmo a intervenção sendo oferecida no horário de expediente dos servidores, a taxa de alcance foi de 14,5\%. Contudo, 59,1\% dos servidores concluíram a intervenção. Outros estudos com o Programa VAMOS também apresentaram taxas inferiores a $50 \%{ }^{20,21}$. Estudo realizado na Atenção Primária à Saúde que avaliou a permanência de idosos no VAMOS verificou que a taxa foi de $51,9 \%$, sendo relacionada ao estado civil dos participantes ${ }^{22}$. Apesar de o perfil dos participantes e contexto serem distintos, entende-se que a logística da intervenção pode ter influenciado na retenção, como a facilidade de acesso, o horário e o local onde a intervenção foi ofertada.

Os servidores, tanto do GI quanto do GC, apresen- 
Tabela 2 - Atividade física habitual de servidores públicos técnico-administrativos pré e pós participação no Programa VAMOS. Florianópolis, $2017(\mathrm{n}=23)$.

\begin{tabular}{|c|c|c|c|c|c|}
\hline \multirow{2}{*}{ Variáveis } & \multicolumn{2}{|c|}{ Média $\pm(\mathrm{dp})$} & \multicolumn{3}{|c|}{ Efeitos } \\
\hline & GI $(n=13)$ & $\mathrm{GC}(\mathrm{n}=10)$ & Grupo & Tempo & Interação \\
\hline $\mathrm{CS}, \mathrm{min} / \mathrm{dia}$ & & & 0,075 & 0,077 & 0,181 \\
\hline Pré-intervenção & $431,27 \pm 88,78$ & $531,11 \pm 115,81$ & & & \\
\hline Pós-intervenção & $419,65 \pm 112,97$ & $452,24 \pm 87,81$ & & & \\
\hline ES & $-0,011$ & $-0,76$ & & & \\
\hline AF leve, $\min /$ dia & & & 0,108 & 0,057 & $0,042^{\#}$ \\
\hline Pré-intervenção & $469,94 \pm 78,05^{\dagger}$ & $367,85 \pm 114,01$ & & & \\
\hline Pós-intervenção & $466,65 \pm 105,92$ & $458,80 \pm 68,91^{*}$ & & & \\
\hline ES & $-0,03$ & 0,96 & & & \\
\hline $\mathrm{AFMV}, \mathrm{min} / \mathrm{dia}$ & & & 0,706 & 0,387 & 0,337 \\
\hline Pré-intervenção & $58,78 \pm 34,11$ & $61,03 \pm 28,85$ & & & \\
\hline Pós-intervenção & $59,43 \pm 27,94$ & $48,96 \pm 26,67$ & & & \\
\hline ES & 0,02 & $-0,43$ & & & \\
\hline AF total, $\mathrm{min} / \mathrm{dia}$ & & & 0,110 & 0,121 & 0,099 \\
\hline Pré-intervenção & $528,73 \pm 88,78$ & $428,89 \pm 115,81$ & & & \\
\hline Pós-intervenção & $526,08 \pm 110,04$ & $507,76 \pm 87,81$ & & & \\
\hline ES & $-0,02$ & 0,76 & & & \\
\hline
\end{tabular}

$\mathrm{GI}=$ grupo intervenção; $\mathrm{GC}=$ grupo controle; $\mathrm{CS}=$ comportamento sedentário; $\mathrm{AF}=$ atividade física; $\mathrm{AFMV}=$ atividade física moderada/ vigorosa; $\mathrm{d}$ = desvio padrão; $\mathrm{n}$ = número de servidores; $\mathrm{p}=$ valor de probabilidade; $\mathrm{ES}$ = tamanho do efeito; valores com significância estatística estão em negrito; $† \mathrm{p} \leq 0,05$ vs. GC; ${ }^{*} \mathrm{p} \leq 0,05$ vs. pré; \# $<$ 0,05 = ANOVA two-way para medidas repetidas.

Tabela 3 - Consumo de alimentos saudáveis e não saudáveis em cinco ou mais dias da semana por servidores públicos técnico-administrativos pré e pós participação no Programa VAMOS. Florianópolis, $2017(\mathrm{n}=23)$.

\begin{tabular}{|c|c|c|c|c|c|}
\hline \multirow{2}{*}{ Variáveis } & \multicolumn{2}{|c|}{$\%(\mathrm{n})$} & \multicolumn{3}{|c|}{ Efeitos } \\
\hline & GI $(n=13)$ & $\mathrm{GC}(\mathrm{n}=10)$ & Grupo & Tempo & Interação \\
\hline \multicolumn{6}{|l|}{ Alimentos saudáveis } \\
\hline Frutas & & & 0,899 & 0,208 & 0,208 \\
\hline Pré-intervenção & $46,2(6)$ & $60,0(6)$ & & & \\
\hline Pós-intervenção & $69,2(9)$ & $60,0(6)$ & & & \\
\hline Verduras/saladas, in natura & & & 0,704 & 0,330 & 0,056 \\
\hline Pré-intervenção & $46,2(6)$ & $60,0(6)$ & & & \\
\hline Pós-intervenção & $76,9(10)$ & $50,0(5)$ & & & \\
\hline Verduras/saladas, cozido & & & 0,056 & 0,767 & $<0,001^{\#}$ \\
\hline Pré-intervenção & $0,0(0)^{\dagger}$ & $70,0(7)$ & & & \\
\hline Pós-intervenção & $46,2(6)^{*}$ & $30,0(3)^{*}$ & & & \\
\hline Feijão & & & 0,354 & 0,782 & 0,782 \\
\hline Pré-intervenção & $15,4(2)$ & $30,0(3)$ & & & \\
\hline Pós-intervenção & $23,1(3)$ & $30,0(3)$ & & & \\
\hline \multicolumn{6}{|l|}{ Alimentos não saudáveis } \\
\hline \multicolumn{6}{|l|}{ Refrigerante } \\
\hline Pré-intervenção & $0,0(0)$ & $0,0(0)$ & - & - & - \\
\hline Pós-intervenção & $0,0(0)$ & $0,0(0)$ & - & - & - \\
\hline Doces & & & 0,835 & 0,375 & 0,375 \\
\hline Pré-intervenção & $30,77(4)$ & $20,0(2)$ & & & \\
\hline Pós-intervenção & $15,38(2)$ & $20,0(2)$ & & & \\
\hline Lanches & & & 0,298 & 0,298 & 0,298 \\
\hline Pré-intervenção & $7,69(1)$ & $0,0(0)$ & & & \\
\hline Pós-intervenção & $0,0(0)$ & $0,0(0)$ & & & \\
\hline
\end{tabular}

$\mathrm{GI}$ = grupo intervenção; $\mathrm{GC}$ = grupo controle; $\mathrm{n}$ = número de participantes; $\%$ = frequência relativa de participantes que consumiram o item alimentar em 5 ou mais dias da semana; $\mathrm{n}=$ número de servidores; $\mathrm{p}=$ valor de probabilidade; ${ }^{\dagger} \mathrm{p} \leq 0,05 \mathrm{vs}$. $\mathrm{GC} ;{ }^{*} \mathrm{p} \leq 0,05$ vs. pré; ${ }^{*} \mathrm{p}<0,05=$ ANOVA two-way para medidas repetidas. 
Tabela 4 - Variáveis antropométricas de servidores públicos técnico-administrativos pré e pós participação no Programa VAMOS. Florianópolis, $2017(\mathrm{n}=23)$.

\begin{tabular}{|c|c|c|c|c|c|}
\hline \multirow{2}{*}{ Variáveis } & \multicolumn{2}{|c|}{ Média \pm dp } & \multicolumn{3}{|c|}{ Efeitos } \\
\hline & GI $(n=13)$ & $\mathrm{GC}(\mathrm{n}=10)$ & Grupo & Tempo & Interação \\
\hline Massa corporal, kg & & & $0,022^{\#}$ & 0,730 & 0,231 \\
\hline Pré-intervenção & $75,48 \pm 14,21$ & $62,21 \pm 7,04$ & & & \\
\hline Pós-intervenção & $74,97 \pm 15,37$ & $63,12 \pm 7,27$ & & & \\
\hline ES & $-0,03$ & 0,12 & & & \\
\hline $\mathrm{IMC}, \mathrm{kg} / \mathrm{m}^{2}$ & & & $0,046^{\#}$ & 0,764 & 0,339 \\
\hline Pré-intervenção & $26,91 \pm 4,70$ & $23,05 \pm 1,36$ & & & \\
\hline Pós-intervenção & $26,77 \pm 5,26$ & $23,33 \pm 1,72$ & & & \\
\hline ES & $-0,03$ & 0,18 & & & \\
\hline $\mathrm{CC}, \mathrm{cm}$ & & & 0,247 & 0,230 & 0,744 \\
\hline Pré-intervenção & $84,92 \pm 15,28$ & $79,05 \pm 4,77$ & & & \\
\hline Pós-intervenção & $84,23 \pm 15,83$ & $77,85 \pm 6,67$ & & & \\
\hline ES & $-0,04$ & $-0,20$ & & & \\
\hline
\end{tabular}

$\mathrm{GI}=$ grupo intervenção; $\mathrm{GC}=$ grupo controle; $\mathrm{IMC}=$ índice de massa corporal; $\mathrm{CC}=$ circunferência da cintura. $\mathrm{Kg}=$ quilogramas; $\mathrm{m}^{2}=$ metros ao quadrado; $\mathrm{cm}$ = centímetros; $\mathrm{dp}=$ desvio padrão; $\mathrm{n}=$ número de servidores; $\mathrm{p}=$ valor de probabilidade; $\mathrm{ES}=$ tamanho do efeito; \# $\mathrm{p}$ $<0,05=$ ANOVA two-way para medidas repetidas.

Tabela 5 - Percepção da qualidade de vida positiva de servidores públicos técnico-administrativos pré e pós participação no Programa VAMOS. Florianópolis, Brasil, 2017 ( $\mathrm{n}=23$ ).

\begin{tabular}{|c|c|c|c|c|c|}
\hline \multirow{2}{*}{ Variável } & \multicolumn{2}{|c|}{$\%(\mathrm{n})$} & \multicolumn{3}{|c|}{ Efeitos } \\
\hline & GI $(n=13)$ & $\mathrm{GC}(\mathrm{n}=10)$ & Grupo & Tempo & Interação \\
\hline QV Positiva & & & $0,026^{\#}$ & $0,048^{\#}$ & $0,048^{\#}$ \\
\hline Pré-intervenção & $69,23(9)^{+}$ & $100,0 \%(10)$ & & & \\
\hline Pós-intervenção & $92,30(12)^{*}$ & $100,0 \%(10)$ & & & \\
\hline
\end{tabular}

$\mathrm{GI}=$ grupo intervenção; $\mathrm{GC}=$ grupo controle; $\mathrm{QV}=$ qualidade de vida; $\%$ = Frequência relativa; $\mathrm{n}=$ número de servidores; $\mathrm{p}=$ valor de probabilidade; $† \mathrm{p} \leq 0,05$ vs. GC; ${ }^{*} \leq 0,05$ vs. pré; $\# \mathrm{p}<0,05=$ ANOVA two-way para medidas repetidas.

taram modesta redução no tempo diário gasto em CS, porém, não foi estatisticamente significante. Esta é uma característica comum na atuação dos servidores públi$\cos ^{9,23}$ e se apresenta como um importante fator de risco para a saúde ${ }^{1}$.Independente da atividade física, o tempo prolongado gasto em CS está associado a vários problemas de saúde ${ }^{9}$. Em outros estudos realizados com o programa VAMOS verificaram que participantes tendem a reduzir pouco o $\mathrm{CS}^{20,21}$ corroborando com os nossos achados. No caso do contexto em questão, a jornada de trabalho se aproxima de oito horas por dia, apesar de ser possível criar estratégias para redução do CS, o tempo sentado é inerente à função desempenhada ${ }^{8}$.

O GI obteve aumento no tempo gasto em AFMV e redução no tempo gasto em AF leve e em AF total. Isso pode ser um indicativo que tenham aumentando a intensidade da $\mathrm{AF}$ de leve que vinham realizando para AFMV. Apesar de evidências apresentarem que a AF leve traz benefícios à saúde ${ }^{24}$, a OMS preconiza que
AFMV traz proteção às $\mathrm{DCNT}^{25}$. Sendo esse, portanto, um resultado satisfatório para o GI. Contrariamente, o GC aumentou de forma estatisticamente significante o tempo gasto em AF leve e em AF total e reduziu o tempo gasto em AFMV. O GC recebeu informações sobre a importância de comportamentos saudáveis antes da sua inserção no estudo, além de seus participantes conviverem com os participantes do GI que, podem ter compartilhado informações impactando na adesão à prática de atividade física. $\mathrm{O}$ apoio social de colegas de trabalho é um importante fator para prática de atividade física, o que reforça que deve ser incorporado nos programas de promoção da saúde no local de trabalho ${ }^{26}$.

Em relação ao comportamento alimentar, os participantes do GI aumentaram o consumo de todos os itens alimentares considerados saudáveis (frutas, verduras/saladas, feijão). O Programa VAMOS utiliza como base para sua intervenção o "Guia Alimentar para a População Brasileira” ${ }^{27}$ e aborda aspectos que vão desde 
a escolha até o preparo dos alimentos, incentivando o aumento no consumo de alimentos in natura/minimamente processados e a redução do consumo de alimentos processados/ultra processados. Em nosso estudo, a maioria dos servidores não tinha o hábito de consumir frutas e verduras. Este também foi o perfil apresentado em outro estudo realizado com servidores de uma instituição pública federal ${ }^{23}$. Outros estudos com o VAMOS também os participantes apresentaram aumento no consumo de alimentos in natura/minimamente processados $^{20,21}$. É importante destacar que o GC reduziu o consumo de alimentos in natura/minimamente processados o que leva a necessidade de intervenções de base educacional junto a estes servidores.

Neste estudo foi possível verificar que os participantes do GI reduziram a massa corporal, o IMC e a circunferência da cintura. As reduções evidenciadas no GI podem se dar pelo fato dos servidores apresentarem níveis maiores de sobrepeso/obesidade no início do estudo, quando comparados ao GC. Entretanto, os participantes do GC aumentaram a medida da massa corporal e o IMC e obtiveram redução somente da circunferência da cintura. A circunferência da cintura está associada diretamente ao CS e ao consumo alimentar ${ }^{28}$. O excesso de gordura corporal é um fator de risco de destaque para o desenvolvimento e agravo de diversas DCNTs e ao aumento de mortalidade, uma vez que predispõe a uma série de riscos cardiovasculares ${ }^{1,29}$.

No Brasil, o excesso de peso é prevalente em mais de $50 \%$ dos servidores e também na população em geral $^{8,23}$. Em estudo realizado na Holanda, a prevalência de excesso de peso foi de $41,5 \%{ }^{30}$ semelhante ao nosso estudo (40\%). Além disso, os servidores apresentam circunferência da cintura aumentada. No Brasil, a prevalência foi entre $45,2 \%{ }^{23}$ e $69 \%{ }^{10}$ semelhante ao nosso estudo, onde $54,3 \%$ dos servidores apresentaram circunferência da cintura aumentada. Tanto o excesso de peso quanto a circunferência da cintura aumentada apresentam relação inversa aos comportamentos saudáveis que são promovidos pelo VAMOS ${ }^{20,21}$.

Em relação a qualidade de vida, foi possível verificar que todos os participantes do GC apresentaram percepção positiva em ambos os momentos do estudo. No GI foi possível observar aumento do número de participantes que passaram a perceber sua qualidade de vida como positiva após participação na intervenção. Inversamente, a saúde negativa está associada a uma maior necessidade de cuidado, afastamento do trabalho por doenças ocupacionais o que acabam prejudicando a produtividade do serviço prestado ${ }^{30}$. Deste modo, o Programa VAMOS se apresenta como fundamental nesse processo, pois estimula a autoconfiança e a autonomia em seus participantes para aderirem e manterem um estilo de vida ativo e saudável, repercutindo na promoção da saúde e consequentemente, aumentando a percepção sob a qualidade de vida ${ }^{12}$.

O presente estudo apresenta algumas limitações. Devido à baixa representatividade amostral, os dados não podem ser generalizados para todo o campo de servidores públicos. $\mathrm{O} \mathrm{GC}$ foi composto a posteriori à composição do GI e os participantes não foram pareados.

Um ponto forte do estudo foi sua originalidade ao propor uma intervenção de mudança comportamental visando a promoção da saúde em servidores de uma universidade pública. $\mathrm{O}$ modelo lógico do VAMOS prevê justamente a inserção da intervenção em diferentes contextos ${ }^{12}$. Outro ponto de destaque foi a mensuração da atividade física habitual e o CS por meio de acelerômetro. Destaca-se ainda a avaliação do estudo por meio da ferramenta RE-AIM. Sua utilização facilita a avaliação e gerenciamento do programa, o que garante uma melhoria constante na intervenção. Por meio dos achados deste estudo e de outros já realizados, o VAMOS foi reformulada e foi desenvolvida uma versão ampliada (3.0), com maior tempo, visando mudanças comportamentais saudáveis mais efetivas e duradouras.

Os servidores participantes do Programa VAMOS apresentaram melhorias no seu estilo de vida por meio de mudanças comportamentais relacionadas a atividade física e alimentação. Desta forma, a intervenção foi efetiva e apresentou potencial para ser ofertada para trabalhadores como estratégia de promoção da saúde. No contexto brasileiro, há necessidade de promover medidas preventivas e educativas direcionadas à saúde do trabalhador, uma vez que elas têm impacto positivo tanto na qualidade de vida como no serviço prestado.

\section{Conflito de interesse}

Os autores declaram não haver conflito de interesse.

\section{Financiamento}

$\mathrm{O}$ estudo foi financiado pela Fundação de Amparo à Pesquisa do Estado de Santa Catarina (FAPESC) por meio do Programa Pesquisa para o SUS: Gestão Compartilhada em Saúde (PPSUS) no 2014TR2263, processo no 3513/2013.

\section{Contribuição dos autores}

Souza PV e Tomicki C, participaram da concepção do manus- 
crito, análise e interpretação dos dados e realizaram a redação do manuscrito. Korand LM, Sandreshi PF, Maciel EC, Ribeiro CG e Rech CR, realizaram a revisão crítica do conteúdo. Benedetti TRB, participou da concepção do programa de mudança de comportamento VAMOS, concepção e redação do projeto deste estudo, busca de financiamento e redação do manuscrito, bem como revisão crítica do conteúdo e foi a idealizadora do projeto.

\section{Agradecimentos}

À Pró-Reitoria de Desenvolvimento e Gestão de Pessoas da Universidade Federal de Santa Catarina por sua colaboração na implementação do Programa VAMOS versão 2.0 aos servidores técnico-administrativos, bem como, aos participantes do estudo.

\section{Referências}

1. Harris RE. Epidemiology of Chronic Disease: global perspectives. 2 ed. Jones \& Bartlet Learning, 2019.

2. Nilson EAF, Andrade RDCS, de Brito DA, de Oliveira ML. Custos atribuíveis a obesidade, hipertensão e diabetes no Sistema Único de Saúde, Brasil, 2018. Rev Panam Salud Publica. 2020;44:e32.

3. Ogata AJN. Promoção da Saúde no Ambiente de Trabalho. Rev Bras Med Trab. 2018;16(Suppl1):1-44.

4. Global Commission on the Future of Work. International Labour Organization. Work for a brighter future. Geneva: ILO, 2019. 78 p.

5. Santi DB, Barbieri AR, Cheade MFM. Absenteísmo-doença no serviço público brasileiro: uma revisão integrativa da literatura. Rev Bras Med Trab. 2018;16(1):71-81.

6. Santa-Marinha MS, Teixeira LR, Maciel EMGS, Moreira MFR. Perfil epidemiológico do absenteísmo-doença na Fundação Oswaldo Cruz no período de 2012 a 2016. Rev Bras Med Trab. 2018;16(4):457-65.

7. Berria J, Petroski Edio L, Minatto G. Excesso de peso, obesidade abdominal e fatores associados em servidores de uma Universidade Federal Brasileira. Rev Bras Cineantropom Desempenho Hum. 2013;15(5):535-50.

8. Gonçalves ECA, Silva DAS, Nunes HEG, Lima TR, Capdeboscq MC, Rinaldi W. Overweight and factors associated in civil servants from Southern Brazil. Rev Bras Cineantropom Desempenho Hum. 2016;18(3):277-86.

9. Biswas A, Oh PI, Faulkner G, Bajaj R, Silver M, Mitchell $\mathrm{M}$ et al. Sedentary Time and Its Association with Risk for Disease Incidence, Mortality, and Hospitalization in Adults. Ann Intern Med. 2015;162(2):123-32.

10. Azevedo ECC, Dias FMRS, Diniz AS, Cabral PC. Consumo alimentar de risco e proteção para as doenças crônicas não transmissíveis e sua associação com a gordura corporal: um estudo com funcionários da área de saúde de uma universidade pública de Recife (PE), Brasil. Ciênc Saúde Col. 2014;19(5):1613-22.

11. Mendes NCF,Matias-Pereira J.Absenteísmo E Contabilidade Pública: Um Estudo Teórico. Revista Eletrônica de Estratégia \& Negócios. 2000;13,156-83.

12. Benedetti T, Manta S, Gomez L, Rech C. Logical model of a behavior change program for community intervention Active Life Improving Health - VAMOS. Rev Bras Ativ Fís Saúde. 2017;22(3):309-13.
13. Borges RA, Tomicki C, Almeida FA, Schwingel A, ChodzkoZajko W, Benedetti TRB. Alcance do programa "VAMOS" na atenção básica-barreiras e facilitadores organizacionais. Rev Bras Geriatr Gerontol. 2019;22(3):e180225.

14. Almeida F, Brito F, Estabrooks P. Modelo RE-AIM: Tradução e Adaptação cultural para o Brasil. Rev. Fam., Ciclos Vida Saúde Contexto Soc. 2013;1(1):6-16.

15. Choi L, Liu Z, Matthews C, Buchowski M. Validation of Accelerometer Wear and Nonwear Time Classification Algorithm. Med Sci Sports Exerc. 2011;43(2):357-64.

16. Freedson P, Melanson E, Sirard J. Calibration of the Computer Science and Applications, Inc. accelerometer. Med Sci Sports Exerc. 1998;30(5):777-81.

17. Silva MC, Ribeiro CG, Benedetti TRB. VAMOS program: instruments for measuring physical activity, feeding and anthropometry. Rev Bras Cineantropom Desempenho Hum. 2020;22:e58256.

18. World Health Organization. Obesity: preventing and managing the global epidemic. Geneva: WHO; 1998.

19. Fleck MPA, Louzada S, Xavier M, Chamovich E, Vieira $\mathrm{G}$, Santos L et al. Aplicação da versão em português do instrumento abreviado de avaliação da qualidade de vida "WHOQOL-bref". Rev Saúde Pública. 2000;34(2),178-83.

20. Gerage AM, Benedetti TRB, Ritti-Dias RM, Dos Santos ACO, Souza BCC, Almeida FA. Effectiveness of a behavior change program on physical activity and eating habits in patients with hypertension: A randomized controlled trial. J Phys Act Health. 2017;14(12):943-52.

21. Meurer ST, Lopes ACS, Almeida FA, Mendonça RDD, Benedetti TRB. Effectiveness of the VAMOS Strategy for Increasing Physical Activity and Healthy Dietary Habits: a randomized controlled community trial. Health Educ. Behav. 2019;46(3):406-16.

22. Konrad LM, Tomicki C, Ribeiro CG, Bezerra JB, Maciel EC, Rech CR et. al. Length of stay in a behavior change program in Primary Health Care: "VAMOS" Program. Rev Bras Ativ Fís Saúde. 2019;24:e0090.

23. Costa MAP, Vasconcelos AGG, Fonseca MJM. Prevalência de obesidade, excesso de peso e obesidade abdominal e associação com prática de atividade física em uma universidade federal. Rev Bras Epidemiol. 2014;17(2):421-36.

24. Ku PW, Hamer M, Liao Y, Hsueh MC, Chen LJ. Devicemeasured light-intensity physical activity and mortality: A meta-analysis. Scand J Med Sci Sports, 2019;30(1),13-24.

25. Organização Mundial da Saúde. Global recommendations on physical activity for health. Genebra: OMS, 2010.

26. Sarkar S, Taylor WC, Lai D, Shegog R, Paxton RJ. Social support for physical activity: Comparison of family, friends, and coworkers. Work. 2016;55(4):893-99.

27. Ministério da Saúde, Secretaria de Atenção à Saúde, Departamento de Atenção Básica. Guia Alimentar para a População Brasileira. Brasília, Brasil: Ministério da Saúde; 2014.

28. Cembranel F, Hallal ALC, González-Chica DA, d'Orsi E. Relação entre consumo alimentar de vitaminas e minerais, índice de massa corporal e circunferência da cintura: um estudo de base populacional com adultos no Sul do Brasil. Cad. saúde pública. 2017;33:e00136616.

29. Tomicki C, Gerage AM, Ritti-Dias RM, Silva DAS, Benedetti TRB. Diagnostic property of anthropometric indicators in the prediction of high body fat estimated by DXA in hypertensive women. Rev Bras Cineantropom Desempenho Hum. 2017;19(3),299-308. 
30. Van der Starre RE, Coffeng JK, Hendriksen IJ, van Mechelen $\mathrm{W}$, Boot CR. Associations between overweight, obesity, health measures and need for recovery in office employees: a cross-sectional analysis. BMC Public Health. 2013;13(1),1-8.

Recebido: 12/06/2019

Aprovado: 17/09/2020

\section{Como citar este artigo:}

Souza PV, Tomicki C, Konrad LM, Sandreshi PF, Ribeiro CG, Maciel EC, Rech CR, Benedetti TRB. Efetividade de uma intervenção na promoção de estilo de vida de servidores públicos. Rev Bras Ativ Fís Saúde. 2020;25:e0135. DOI: 10.12820/rbafs.25e0135 